\title{
CONNECTED MAPPINGS OF HAUSDORFF SPACES
}

WILLIAM J. PERVIN AND NORMAN LEVINE

1. Introduction. A fundamental property of continuous mappings is that the image of a connected set is a connected set. This property will be taken as the definition of connected mappings. We are naturally led to two lines of research concerning connected mappings. Since the requirements for a set to be connected are quite strong, we might expect that connected mappings would have interesting topological properties of their own. Furthermore, since continuous mappings are special cases of connected mappings, we would like to know what other conditions must be placed upon the mapping or upon the spaces in order to be able to conclude that a connected mapping is continuous. Both of these lines will be pursued in this paper.

\section{Preliminaries.}

Definition 2.1. Let $K$ and $K^{*}$ be Hausdorff spaces and $f$ a singlevalued mapping of $K$ into $K^{*}$. The mapping $f$ is said to be connected if and only if the image of every connected subset of $K$ is a connected subset of $K^{*}$. The reader will easily construct examples of connected mappings which are not continuous. The following, however, will be useful in the sequel. Let $w$ be the mapping of the unit interval $I=[0,1]$ of real numbers defined as follows $[3$, p. $82 ; 9$, p. 202]: $w(x)=\lim \sup \left(a_{1}+a_{2}+\cdots+a_{n}\right) / n$ for each $x \in I$ where the $a_{i}$ are given by the unique nonterminating binary expansion of the number $x=\left(0 \cdot a_{1} a_{2} a_{3} \cdots\right)$. This mapping takes on every value between 0 and 1 in every interval; hence it is a connected mapping, but it is not continuous.

THEOREM 2.1. Necessary and sufficient conditions for the continuity of a real-valued mapping $f$ of a real variable defined in a closed interval $[a, b]$ are the following: (i) If $x_{1}$ and $x_{2}$ are any two points of the interval, $f(x)$ takes on each value between $f\left(x_{1}\right)$ and $f\left(x_{2}\right)$ in the interval $\left[x_{1}, x_{2}\right]$; (ii) For every value of $y$, the set of points $\{x: f(x)=y\}$ is closed [6].

Condition (i) of Theorem 2.1 is known as Darboux's condition and is well known in the theory of functions of a real variable.

THEOREM 2.2. Suppose $g$ is a real valued mapping of the unit interval $I=[0,1]$. If the mapping $g$ is of the first Baire class and satisfies Darboux's condition, then the graph of $g$ (i.e., the set of points $(x, y)$ in

Received by the editors May 2, 1957. 
the plane such that $y=g(x))$ is connected [4, p. 304]. Furthermore, if the derivative $g^{\prime}$ is finite for all values of $x$, then the graph of $g^{\prime}$ is connected [2, p. 383].

TheOREM 2.3. Suppose $f$ is a connected mapping of the metric space $M$ onto the metric space $f(M)$. For any point $p$ in $M$ the assertion that $M$ is locally connected at $p$ is equivalent to saying that every real-valued map $f$ on $M$, having the property that $f^{-1}(q)$ is closed for each point $q \in f(M)$, is continuous at $p[\mathbf{1}]$.

Definition 2.2. Let $K$ and $K^{*}$ be Hausdorff spaces and $f$ a singlevalued mapping of $K$ onto $K^{*}$. The mapping $f$ is said to be biconnected if and only if it is $1-1, f$ is connected, and $f^{-1}$ is connected.

Theorem 2.4. Suppose $M$ and $M^{*}$ are separable metric spaces and $f$ is a biconnected mapping of $M$ onto $M^{*}$. If $M^{*}$ is semi-locallyconnected, $f$ is continuous. Clearly, if $M$ and $M^{*}$ are both semi-locallyconnected, $f$ is a homeomorphism. If $C$ is a connected subset of $M$, then $f(\bar{C}) \subset \mathrm{Cl}(f(C))$. If $M^{*}$ is compact, then every subcontinuum of $M$ is transformed into a subcontinuum of $M^{*}[7]$.

\section{Properties of connected mappings.}

Definition 3.1. If $K$ and $K^{*}$ are two Hausdorff spaces and $f$ is a mapping of $K$ into $K^{*}$, then $f$ is called monotone if and only if for every $p^{*}$ in $K^{*}, f^{-1}\left(p^{*}\right)$ is a connected subset of $K$.

THEOREM 3.1. If $f$ is a monotone connected mapping of $K$ into $K^{*}$, then for every $p^{*}$ in $K^{*}, f^{-1}\left(p^{*}\right)$ is a closed subset of $K$.

Proof. Suppose $f^{-1}\left(p^{*}\right)$ is not closed for some $p^{*}$ in $K^{*}$. Let $p$ be a limit point of $f^{-1}\left(p^{*}\right)$ which does not belong to $f^{-1}\left(p^{*}\right)=A$. But $A$ is a connected subset of $K$ since $f$ is monotone. Now $f(A)=p^{*}$. Consider the set $A \cup_{p}$. It is a connected subset of $K$. Therefore $f\left(A \cup_{p}\right)$ is a connected subset of $K^{*}$. But $f(A \cup p)=f(A) \cup f(p)=p^{*} \cup f(p)$. A connected subset of a Hausdorff space cannot consist of two distinct points and hence $f(p)=p^{*}$. Hence $p$ is in $f^{-1}\left(p^{*}\right)$, a contradiction.

In view of Theorem 3.1, the above Theorem 2.3 of Klee and Utz may now be stated thus: Every monotone connected mapping of the metric space $M$ to the real numbers is continuous at a point $p$ in $M$ if and only if $M$ is locally connected at $p$.

TheOREM 3.2. A monotone real-valued connected mapping of a real variable is continuous.

Proof. By the above Theorem 2.1 of Rowe, a necessary and sufficient condition for a real-valued connected mapping $f$ of a real vari- 
able to be continuous is that for every $y$, the set $f^{-1}(y)$ is closed. But by Theorem 3.1, $f^{-1}(y)$ is closed, and hence $f$ is continuous.

It is well known that a necessary and sufficient condition that a mapping $f$ from $K$ to $K^{*}$ be continuous is that for every subset $E$ of $K, f(\bar{E}) \subset C l(f(E))$. Analogous to this theorem we have:

THEOREM 3.3. Let $f$ be a connected mapping of the Hausdorff space $K$ into the Hausdorff space $K^{*}$. If $C$ is a connected subset of $K$, then $f(\bar{C}) \subset \mathrm{Cl}(f(C))$.

Proof. Let $p^{*} \in f(\bar{C})$. Since $p^{*} \in f(\bar{C})$, there is a point $p \in \bar{C}$ such that $f(p)=p^{*}$. Case 1: $p \in C$. Then $p^{*}=f(p) \in f(C)$, and the theorem follows. Case 2: $p \in \bar{C}-C$. Then $p$ is a limit point of $C$, and since $C \subset C \cup_{p} \subset \bar{C}$, we have $C \cup_{p}$ a connected set. Hence $f\left(C \cup_{p}\right)=f(C)$ $\cup f(p)=f(C) \cup_{p^{*}}$ is a connected set since $f$ is a connected mapping. But every connected set containing more than one point is dense-initself, and so $p^{*}$ must be a limit point of $f(C)$ unless $f(C)$ is a single point in which case $p^{*}=f(C)$. In either case, $p^{*} \in \operatorname{Cl}(f(C))$.

It is interesting to note that connected mappings may not even be measurable. Let $Q$ be the nonmeasurable set in the unit interval described by Munroe [5, pp. 142-143]. If two points in the unit interval differ by a rational number, then they cannot both lie in $Q$. Now let us define a function $f$ in terms of $w$ introduced previously. For $x$ in $Q$, let $f(x)=1$; for $x$ not in $Q$, if $w(x) \neq 1$ let $f(x)=w(x)$ but if $w(x)=1$ let $f(x)=0$. Clearly the set of points on which $f(x)=1$ is just $Q$ which is nonmeasurable, and hence $f$ is nonmeasurable. Now we will show that $f$ is a connected mapping. If $f$ is not connected, there is an interval $J$ in the unit interval such that $f(J)$ is not connected. Then we have three numbers $a<y<b$ such that $a$ and $b$ belong to $f(J)$ but $y$ does not. We cannot have $y=1$ since $b \leqq 1$. In order for $y$ not to be in $f(J)$ we must have all points $x$ in $J$ for which $w(x)=y$ belong to $Q$. Choose one of these values of $x$ in the interior of $J$ and call it $p$. This is possible since such values of $x$ are dense in the unit interval by the definition of $w$. Since $p$ is in the interior of $J$, let $\epsilon$ denote the distance from $p$ to the right hand endpoint of $J$. Choose $n$ so that $(1 / 2)^{n}<\epsilon$. Then consider the point $p^{*}=p+(1 / 2)^{n}$. This point lies in $J$, and in its binary expansion all digits from the $(n+1)$ st on are the same as in the binary expansion for $p$; hence $w\left(p^{*}\right)=w(p)=y$. But both $p$ and $p^{*}$ cannot be in $Q$ since their difference is $(1 / 2)^{n}$, a rational number. Hence there must be some point in $J$ whose image is $y$, and therefore $f$ is a connected mapping.

THEOREM 3.4. Let $K_{1}, K_{2}$, and $K_{3}$ be Hausdorff spaces and $f$ a mapping of $K_{1} \times K_{2}$ into $K_{3}$. If $f$ is a connected mapping, then $f$ has the 
following properties: (i) $f(x, B)$ is connected for all $x$ in $K_{1}$ and all $B$ connected in $K_{2}$; (ii) $f(A, y)$ is connected for all $A$ connected in $K_{1}$ and all $y$ in $K_{2}$.

The reader will find this theorem easy to verify. The converse to Theorem 3.4 is not true as shown by the well known example $f(x, y)$ $=x y /\left(x^{2}+y^{2}\right)$ if both $x$ and $y$ are not 0 and $f(0,0)=0[8$, p. 193]. This function is continuous in each variable separately and hence connected in each variable separately. The diagonal $y=x$ is a connected subset of the plane and on it $f(x, x)=1 / 2$ if $x \neq 0$ while $f(0,0)$ $=0$. Hence this function is not a connected mapping. Theorem 3.4 does, however, have a partial converse in

THEOREM 3.5. Let $f$ be a mapping of $K_{1} \times K_{2}$ into $K_{3}$. Suppose $f$ has the properties (i) and (ii) of Theorem 3.4. Then $f(A, B)$ is connected for all $A$ connected in $K_{1}$ and all $B$ connected in $K_{2}$.

We omit the proof. In this connection there exists a real-valued mapping of two real variables which is continuous in each variable separately and connected but not continuous. The mapping is

$$
f(x, y)=\frac{2 x y}{x^{2}+y^{2}} \sin \frac{\pi}{\left(x^{2}+y^{2}\right)^{1 / 2}}
$$

if $x$ and $y$ are not both 0 and $f(0,0)=0$. The details are left to the reader.

In the sequel we shall give conditions that either the mapping or the spaces must satisfy in order to be able to conclude that a connected mapping is continuous.

Definition 3.2. A mapping $f$ of a Hausdorff space $K$ into a Hausdorff space $K^{*}$ is said to have at worst a removable discontinuity at a point $p$ in $K$ if and only if for every sequence of points $p_{n}$ different from $p$ such that $\lim p_{n}=p$, we have $\lim f\left(p_{n}\right)$ exists.

It is clear that if a mapping $f$ has at worst a removable discontinuity at a point $p$, then $\lim f\left(p_{n}\right)$ is unique for every sequence of points in $K$ whose elements are different from $p$. We now give an example of a real-valued connected mapping $f$ of a complete metric space $M$ on which $f$ has at worst removable discontinuities at every point, but for some points $p$ in $M, f(p)$ does not equal the unique $\lim f\left(p_{n}\right)$ and hence $f$ is not continuous. Let $M$ be the set of real numbers consisting of the point 0 and all points of the form $1 / n$ for $n=1,2,3, \cdots$. Let $f$ be defined as follows: $f(p)=1$ if $p \neq 0$ and $f(0)=0$. This mapping is continuous on $M-0$. Furthermore, $f$ is connected since the only connected subsets of $M$ are single points. At $0, f$ has at worst a removable discontinuity and $\lim f\left(p_{n}\right)=1$. But 
$f(0)=0$, and hence $f$ is discontinuous at 0 . Of course the fact that $f$ is connected does not have any significance in the problem of obtaining conditions necessary for continuity since the only connected subsets of $M$ are single points. In order to assure that we have some open connected sets in $M$ we will usually assume that $M$ is locally connected. Furthermore, in order to be able to use the sequential property of continuity we will assume that $M$ is a first axiom space.

THEOREM 3.6. Let $f$ be a connected mapping of the locally connected first axiom Hausdorff space $K$ into $K^{*}$. Then $f$ is continuous at $p$ if and only if $f$ has at worst a removable discontinuity at $p$.

Proof. Suppose that $f$ is connected and has at worst a removable discontinuity at $p$ in $K$. Let $p^{*}$ denote the unique $\lim f\left(p_{n}\right)$ for every sequence of points in $K$ converging to $p$ but different from $p$. Suppose $f(p) \neq p^{*}$. There exist open sets $O_{1}^{*}$ and $O_{2}^{*}$ such that $f(p) \in O_{1}^{*}$, $p^{*} \in O_{2}^{*}$, and $O_{1}^{*} \cap O_{2}^{*}=\varnothing$ by the separation axiom for Hausdorff spaces. Since $K$ is the first axiom, there exists an open set $O$ such that $p \in O$ and for $p^{\prime} \in O-p$ we have $f\left(p^{\prime}\right) \in O_{2}^{*}$. But since $O_{1}^{*} \cap O_{2}{ }^{*}$ $=\varnothing$, for $p^{\prime}$ in $O-p$ we have $f\left(p^{\prime}\right) \in O_{1}^{*}$. Since $K$ is locally connected, there exists a connected open set $C \subset O$ containing $p$. Then $f(C)$ is a connected set in $K^{*}$ since $f$ is connected and $f(p)$ is in $f(C)$. But $f(C)$ can have no other point in $O_{1}^{*}$ since for $p^{\prime}$ in $O-p, f\left(p^{\prime}\right) \notin O_{1}^{*}$. Hence $f(p)$ is not a limit point of $f(C)$, and hence $f(C)$ is not dense-initself. Since $f(C)$ is connected it must contain only the one point $f(p)$. This implies that $f$ is continuous at $p$. Since a continuous mapping is connected and has no discontinuities, the conditions given must be both necessary and sufficient.

Definition 3.3. Let $f$ be a mapping of the Hausdorff spaces $K$ into the Hausdorff space $K^{*}$. For every point $p$ in $K$ let the set of limit points of $f$ at $p$, denoted by $L(f ; p)$, be the set of all points $p^{*}$ in $K^{*}$ for which there exists a sequence $p_{n}$ of points in $K$ such that $\lim p_{n}=p$ and $\lim f\left(p_{n}\right)=p^{*}$.

Lemma 3.1. Suppose $f$ is a mapping of the first axiom space $K$ into the first axiom space $K^{*}$. For every point $p$ in $K, L(f ; p)$ is a closed subset of $K^{*}$.

Proof. Let $p$ be any point in $K$ and suppose $p^{*}$ is a limit point of $L(f ; p)$. Since $K$ is first axiom, let $\left\{O_{n}\right\}$ be a sequence of open sets containing $p$ such that for every open set $O$ containing $p$, there exists an index $N$ such that $O_{n} \subset O$ for all $n \geqq N$. We can assume the $O_{n}$ to be monotone descending. Let $\left\{O_{n}^{*}\right\}$ be a monotone sequence of open sets in $K^{*}$ containing $p^{*}$ such that for any open set $O^{*}$ containing 
$p^{*}$, there exists an index $N^{\prime}$ such that $O_{n}^{*} \subset O^{*}$ for all $n \geqq N^{\prime}$.

Consider $O_{j}^{*}$ for some fixed index $j$. Since $p^{*}$ is a limit point of $L(f ; p)$, there must be some point $q^{*}$ in $O_{j}^{*} \cap L(f ; p)-p^{*}$. But since $q^{*}$ is in $L(f ; p)$, there exists a sequence $p_{n}$ of points in $K$ such that $\lim p_{n}=p$ and $\lim f\left(p_{n}\right)=q^{*}$. Now since $\lim f\left(p_{n}\right)=q^{*}$ and $O_{j}^{*}$ is an open set containing $q^{*}$, there is an index $N_{1}$ such that $f\left(p_{n}\right) \in O_{j}^{*}$ for $n \geqq N_{1}$. Furthermore, since $\lim p_{n}=p$ and $O_{j}$ is an open set containing $p$, there is an index $N_{2}$ such that $p_{n} \in O_{j}$ for all $n \geqq N_{2}$. Pick $N$ $=\max \left(N_{1}, N_{2}\right)$ and relabel the point $p_{N}$ thus chosen as $p_{j}$ corresponding to the fixed $j$ chosen above. This point $p_{j}$ now has the properties that it belongs to $O_{j}$ and also has $f\left(p_{j}\right)$ in $O_{j}^{*}$. This we do for each $j$, giving a sequence of points $p_{j}$ in $K$. We assert that $\lim p_{j}$ $=p$. The details are easy, and we leave them for the reader to verify. Hence $L(f ; p)$ is a closed set.

THEOREM 3.7. If $f$ is a connected mapping of the locally connected first axiom space $K$ into the compact first axiom space $K^{*}$, then $L(f ; p)$ is a connected subset of $K^{*}$ for every $p$ in $K$.

Proof. Suppose $L(f ; p)$ is disconnected for some $p$ in $K$. Then let $L(f ; p)=A^{*} \mid B^{*}$ be a separation. By the preceding lemma $L(f ; p)$ is closed, and hence $A^{*}$ and $B^{*}$ are closed subsets of $K^{*}$. But $K^{*}$ is compact and hence normal. Therefore there exist two disjoint open sets $O_{1}^{*}$ and $O_{2}^{*}$ such that $A^{*} \subset O_{1}^{*}$ and $B^{*} \subset O_{2}^{*}$. Thus $L(f ; p) \subset O_{1}^{*} \cup O_{2}^{*}$. Suppose that for every open set $O$ containing $p$ there exists a point $p^{\prime}$ in $O$ such that $f\left(p^{\prime}\right) \in K^{*}-\left(O_{1}^{*} \cup O_{2}^{*}\right)$; this will lead to a contradiction. Since $K$ is first axiom we can take $\left\{O_{j}\right\}$ a monotone descending sequence of open sets containing $p$ as in the above lemma. By the same argument used in the lemma we may construct a sequence $\left\{p_{n}\right\}$ of points in $K$ such that $\lim p_{n}=p$. Consider the sequence $\left\{f\left(p_{n}\right)\right\}$ in $K^{*}$. Since $K^{*}-\left(O_{1}^{*} \cup O_{2}^{*}\right)$ is a closed subset of the compact space $K^{*}$, it is also compact. Hence the sequence $\left\{f\left(p_{n}\right)\right\}$ of points in $K^{*}-\left(O_{1}^{*} \cup O_{2}^{*}\right)$ has a convergent subsequence which converges to some $p^{*}$ in $K^{*}-\left(O_{1}^{*} \cup O_{2}^{*}\right)$. But then $p^{*}$ is in $L(f ; p)$, and this contradicts the fact that $L(f ; p) \subset O_{1}^{*} \cup O_{2}^{*}$. Therefore there is some open set $O$ containing $p$ such that $f(O) \subset O_{1}^{*} \cup O_{2}^{*}$. Since $K$ is locally connected, there exists a connected open set $C \subset O$ containing $p$ and such that $f(C) \subset O_{1}^{*} \cup O_{2}^{*}$. But since $f$ is connected, $f(C)$ is connected in $K^{*}$ and $f(C)$ lies completely in $O_{1}^{*}$ or $O_{2}^{*}$. But if $f(C)$ lies in one of these op'n sets, the other can have no points of $L(f ; p)$ in it, and so either $A^{*}$ or $B^{*}$ must be empty. Thus $L(f ; p)$ is connected. Of course $L(f ; p)$ is never empty since $f(p)$ always lies in it.

THEOREM 3.8. Suppose $f$ is a connected mapping of the locally con- 
nected first axiom space $K$ into the compact first axiom space $K^{*}$. The mapping $f$ is continuous at $p$ in $K$ if and only if $L(f ; p)$ is finite or denumerable.

Proof. Since a continuous mapping is connected and has $L(f ; p)$ $=f(p)$, we need only prove that the conditions are sufficient to make $f$ continuous. Now $L(f ; p)$ is connected, and $f(p)$ is in $L(f ; p)$. Furthermore, $K^{*}$ is compact, and therefore $L(f ; p)$ either consists of one point or it is nondenumerable. But by assumption it is not nondenumerable, and so $L(f ; p)=f(p)$. Hence $f$ is continuous at $p$.

The following theorems are generalizations of the results of Theorem 2.4 of Tanaka from separable metric spaces to Hausdorff spaces.

THEOREM 3.9. Let $f$ be a biconnected mapping of the Hausdorff space $K$ onto the semi-locally-connected Hausdorff space $K^{*}$, then $f$ is continuous.

Proof. Let $O^{*}$ be an open set in $K^{*}$. We shall show that $f^{-1}\left(O^{*}\right)$ is open in $K$. Take $p$ in $f^{-1}\left(O^{*}\right)$. Let $f(p)=p^{*} \in O^{*}$. Since $K^{*}$ is semilocally-connected, there exists an open set $O_{0}^{*}$ contained in $O^{*}$ such that $p^{*}$ is in $O_{o}^{*}$ and $K^{*}-O_{o}^{*}$ consists of a finite number of components. Let these components be $C_{1}^{*}, C_{2}^{*}, \cdots, C_{n}^{*}$. Thus $O_{o}^{*}$ $=K^{*}-\bigcup_{i=1}^{n} C_{i}^{*}$. Now each $C_{i}^{*}$ is connected and closed. Let $C_{i}$ $=f^{-1}\left(C_{i}^{*}\right)$ for each $i$. The sets $C_{i}$ are connected since $f$ is biconnected. Now suppose $p$ belongs to $\bar{C}_{j}$ for some $j$. Then $C_{j} \cup p$ would be connected, and since $f$ is biconnected $f\left(C_{j} \cup p\right)=f\left(C_{j}\right) \cup f(p)=C_{j}^{*} \cup p^{*}$ is connected. If this connected set consists of only one point, we would have $p^{*}=C_{j}^{*}$. If this connected set contains more than one point, it is dense-in-itself. In that case $p^{*}$ would have to be a limit point of $C_{j}^{*}$ But $C_{j}^{*}$ is closed, and this would imply that $p^{*}$ is in $C_{j}^{*}$. But both $p^{*}$ in $C_{j}^{*}$ and $p^{*}=C_{j}^{*}$ contradict the fact that $C_{j}^{*}$ is a component of the complement of $O_{o}^{*}$, an open set containing $p^{*}$. Therefore $p$ belongs to no $\mathrm{Cl}\left(C_{j}\right)$. Hence there exists an open set $O_{i}=K-\mathrm{Cl}\left(C_{i}\right)$ such that $p \in O_{i}=K-\mathrm{Cl}\left(C_{i}\right)$ for each $i$. Let $O_{p}=\bigcap_{i=1}^{n} O_{i}$. Then $O_{p}$ is an open set in $K$ since it is the intersection of a finite number of open sets. Also $p \in O_{p}$. We note that since $f\left(C_{i}\right)=C_{i}^{*}, f\left(\mathrm{Cl}\left(C_{i}\right)\right) \supset C_{i}^{*}$ and so $K^{*}-f\left(\mathrm{Cl}\left(C_{i}\right)\right) \subset K^{*}-C_{i}^{*}$. Now $f$ is $1-1$, and hence

$$
\begin{aligned}
f\left(O_{p}\right)= & f\left(\bigcap_{i=1}^{n} O_{i}\right)=\bigcap_{i=1}^{n} f\left(O_{i}\right)=\bigcap_{i=1}^{n} f\left(K-\bar{C}_{i}\right)=\bigcap_{i=1}^{n}\left[K^{*}-f\left(\mathrm{Cl}\left(C_{i}\right)\right)\right] \\
& \subset \bigcap_{i=1}^{n}\left(K^{*}-C_{i}^{*}\right)=K^{*}-\bigcup_{i=1}^{n} C_{i}^{*}=O_{o}^{*} .
\end{aligned}
$$


Since $f\left(O_{p}\right) \subset O_{o}^{*}, f$ is continuous.

Hence if $K$ and $K^{*}$ are both semi-locally-connected Hausdorff spaces, every biconnected mapping of $K$ and $K^{*}$ is a homeomorphism.

THEOREM 3.10. Let $f$ be a biconnected mapping of $K$ onto $K^{*}$ where $K$ and $K^{*}$ are locally connected compact Hausdorff spaces, then $f$ is a homeomorphism.

Proof. Let $O^{*}$ be an open set in $K^{*}$. Let $p$ be in $f^{-1}\left(O^{*}\right)$. Let $f(p)=p^{*} \in O^{*}$. Since $K^{*}$ is locally connected, there is a connected open set $C^{*}$ such that $p^{*} \in C^{*} \subset O^{*}$. Then $p \in f^{-1}\left(C^{*}\right)$. For each point $p_{\alpha}^{*}$ in $K^{*}-C^{*}$ let $U_{\alpha}^{*}$ be an open set containing $p_{\alpha}^{*}$ but not $p^{*}$. Since $K^{*}$ is compact, there is an open set $V_{\alpha}^{*}$ such that $p_{\alpha}^{*} \in V_{\alpha}^{*} \subset \mathrm{Cl}\left(V_{\alpha}^{*}\right)$ $\subset U_{\alpha}^{*}$. Finally, since $K^{*}$ is locally connected, there is a connected set $O_{\alpha}^{*}$ such that $p_{\alpha}^{*} \in O_{\alpha}^{*} \subset V_{\alpha}^{*}$. Thus $O_{\alpha}^{*}$ is a connected open set containing $p_{\alpha}^{*}$ whose closure does not contain $p^{*}$. Then $U_{\alpha} O_{\alpha}^{*}$ is an open covering for $K^{*}-C^{*}$. By compactness, $K^{*}-C^{*} \subset \bigcup_{i=1}^{n} O_{i}^{*}$. Let $O_{i}$ $=f^{-1}\left(O_{i}^{*}\right)$ for each $i$. Then $p \notin O_{i}$ for any $i$ since $p^{*} \notin O_{i}^{*}$ for any $i$.

Now suppose for some $j, p \in \operatorname{Cl}\left(O_{j}\right)$. Since $O_{j}$ is connected, $O_{j} \cup_{p}$ is connected. But $f\left(O_{j} \cup p\right)=f\left(O_{j}\right) \cup f(p)=O_{j}^{*} \cup_{p}$. Hence $O_{j}^{*} \cup p^{*}$ is connected. If this connected set consists of one point, we have $p^{*}=O_{j}^{*}$. If it contains more than one point, it is dense-in-itself and $p^{*}$ is a limit point of $O_{j}^{*}$. In either case we have $p^{*} \in \operatorname{Cl}\left(O_{j}^{*}\right)$ which contradicts the definition of the sets $O_{\alpha}^{*}$. Therefore $p \notin \bar{O}_{i}$ for every $i$. Therefore for each index $i$, there exists an open set $U_{i}$ such that $p \in U_{i}=K-\operatorname{Cl}\left(O_{i}\right)$. Let $O_{p}=\bigcap_{i=1}^{n} U_{i}$. Then $O_{p}$ is an open set containing $p$. We note that $f\left(O_{i}\right)=O_{i}^{*}$, so $f\left(\mathrm{Cl}\left(O_{i}\right)\right) \supset O_{i}^{*}$, and hence $K^{*}-f\left(\mathrm{Cl}\left(O_{i}\right)\right) \subset K^{*}-O_{i}^{*}$. Now $f$ is $1-1$ and hence

$$
\begin{aligned}
f\left(O_{p}\right) & =f\left(\bigcap_{i=1}^{n} U_{i}\right)=\bigcap_{i=1}^{n} f\left(U_{i}\right)=\bigcap_{i=1}^{n} f\left(K-\mathrm{Cl}\left(O_{i}\right)\right) \\
& =\bigcap_{i=1}^{n}\left[K^{*}-f\left(\mathrm{Cl}\left(O_{i}\right)\right)\right] \subset \bigcap_{i=1}^{n}\left(K^{*}-O_{i}^{*}\right)=K^{*}-\bigcup_{i=1}^{n} O_{i}^{*} \subset C^{*} \subset O^{*} .
\end{aligned}
$$

Since $f\left(O_{p}\right) \subset O^{*}, f$ is continuous. By symmetry, $f^{-1}$ is also continuous and so $f$ is a homeomorphism.

\section{BIBLIOGRAPHY}

1. V. L. Klee and W. R. Utz, Some remarks on continuous transformations, Proc. Amer. Math. Soc. vol. 5 (1954) pp. 182-184.

2. B. Knaster and C. Kuratowski, Sur quelques propriétés topologiques des fonctions derivées, Rend. Circ. Mat. Palermo vol. 49 (1925) pp. 382-386.

3. C. Kuratowski, Topologie II, Warsaw, 1952. 
4. C. Kuratowski and W. Sierpinski, Les fonctions de classe 1 et les ensembles connexes ponctiformes, Fund. Math. vol. 3 (1922) pp. 303-313.

5. M. E. Munroe, Measure and integration, Cambridge, Addison-Wesley, 1953.

6. C. H. Rowe, Note on a pair of properties which characterize continuous functions, Bull. Amer. Math. Soc. vol. 32 (1926) pp. 285-287.

7. T. Tanaka, On the family of connected subsets and topology of spaces, Journal of the Mathematical Society of Japan, vol. 7, 1955.

8. R. Vaidyanathaswamy, Treatise on set topology, Madras, 1947.

9. L. Vietoris, Stetige Mengen, Monatschefte fur Mathematik und Physik vol. 31 (1921) pp. 173-204.

10. O. H. Hamilton, Fixed points for certain noncontinuous transformations, Proc. Amer. Math. Soc. vol. 8 (1957) pp. 750-756.

University of Pittsburgh

\title{
A MULTIPLICATION THEOREM FOR POSITIVE REAL FUNCTIONS ${ }^{1}$
}

\author{
F. M. REZA ${ }^{2}$
}

Modern network synthesis relies heavily on the use of Positive Real Functions. A function $Z(s)$ of the complex frequency $s$ is said to be a P.R.F. (short for Positive Real Function) when it satisfies the following three conditions.

(a) $Z(s)$ is analytic and single valued for $\operatorname{Re} s \geqq 0$ except possibly for poles on the imaginary axis,

(b) $Z(s)$ is real for real $s$,

(c) $\operatorname{Re} Z(s) \geqq 0$ for $\operatorname{Re} s \geqq 0$.

When in addition $\operatorname{Re} Z(i y)=0$, the function $Z(s)$ will be called an I.P.R.F.

The object of this note is to contribute two theorems clarifying the "algebra" of Positive Real Functions, and providing a key for their generation. These theorems employ a simple normalization procedure (operation $N$ ) after which the product of any number of normalized P.R.F.'s is a normalized P.R.F. Since obviously the sum of several P.R.F.'s is a P.R.F. then one will obtain a rather simple algebra for these functions. One may symbolically write:

Received by the editors May 20, 1957 and, in revised form, June 26, 1957 and November 2, 1957.

1 This paper was written in the course of research conducted under the sponsorship of the Office of Ordnance Research, U. S. Army.

2 This paper was presented to the International Scientific Radio Union's twelf th General Assembly, Boulder, Colorado, 1957. 